\title{
Insight into cardiovascular risk factors in patients with acromegaly
}

\author{
Maria Fleseriu
}

Received: 11 December 2013/Accepted: 22 December 2013/Published online: 11 January 2014

(C) Springer Science+Business Media New York 2014

In this issue of Endocrine, Dr. Ragonese and colleagues provide a well-written, extensive study on the prediction of ischemic cardiovascular events (ICE) in acromegalic patients stratified by Framingham score (FS) and Agatston score (AS) [1]. FS is based on conventional risk factors, such as gender, age, hypertension, smoking habits, diabetes mellitus, and lipid status, while AS is calculated by the measurement of calcium burden in the main coronary arteries by electron beam computed tomography.

The strength of this study [1] is represented by the extensive cardiac workup in 52 acromegalic patients with controlled and uncontrolled disease followed for 5 years. Interestingly, an AS $>400$, but not a high FS at entry, was associated with an increased risk of lethal ICE, but the total number of lethal ICE was low.

Rather than summarizing study-related findings, I will comment on current and somewhat limited understanding of cardiovascular risk factors in patients with acromegaly. Increased mortality rate is primarily due to cardiovascular disease [2-5]. However, what is often controversial is whether growth hormone $(\mathrm{GH})$ excess has deleterious effects per se on the cardiovascular system or whether cardiovascular disease results from the frequently observed increased prevalence of associated risk factors. Although mortality seems to decrease, improvement or reversal of cardiovascular morbidity by effective GH control also remains controversial. It is also important to determine which patients are at higher risk of lethal ICE.

\footnotetext{
M. Fleseriu ( $\square$ )

Northwest Pituitary Center, Departments of Medicine and Neurological Surgery, Oregon Health \& Science University, 3181 SW Sam Jackson Park Road (BTE 472), Portland, OR 97239, USA

e-mail: fleseriu@ohsu.edu
}

Cardiomyopathy of acromegaly [4] includes both structural and functional abnormalities: concentric biventricular hypertrophy (20\% in young patients and in up to $90 \%$ of patients with long-standing disease) and diastolic dysfunction [6], leading to the development of heart failure with preserved ejection fraction. However, the clinical syndrome of heart failure is relatively uncommon (1-10\%). Is acromegalic cardiomyopathy reversible? Suppression of GH and insulinlike growth factor 1 (IGF-1) could improve diastolic function [3]; however, systolic function and exercise tolerance response are variable and dependent mostly on disease duration and the presence of hypertension and diabetes. As expected, younger patients respond better to treatment than middle-aged patients. Likewise, ventricular remodeling increases risk of mitral, aortic, and tricuspid regurgitation in the late stages of acromegaly [3]. Arrhythmias (atrial fibrillation, supraventricular tachycardia, and ventricular arrhythmias), due to phenotypic changes in membrane proteins, conduction system, and uncoupling of cardiac myocytes with increased re-entry events, and autonomic dysfunction [7] are also common.

The development of atherosclerotic disease seems more "complicated": Hypertension and diabetes are by far the main predisposing factors, and up to half of patients could be at intermediate-to-high risk for coronary artery disease (CAD). GH and IGF-1 cause direct endothelial dysfunction, which decreases vasodilation and could increase risk of future ICE. Despite this unfavorable cardiovascular risk profile, it is not clear whether acromegalic patients have indeed an increased risk of CAD per se. Mouse models, for example, suggested that the excess mortality might be due to the development of hypertrophic cardiomyopathy rather than increased rates of atherosclerotic CAD.

Growth hormone excess (which could predict the increase in low-density lipoprotein cholesterol (LDL-C), 
oxidized LDL, and endothelin-1) contributes to the development of insulin resistance [8]; interaction between them could be responsible for the appearance of atherogenic prooxidative and pro-inflammatory factors, including endothelial dysfunction [9].

Additionally, multiple studies report early vascular alterations, mainly represented by impaired flow-mediated dilation of brachial artery, increased intima-media-thickness (IMT) of the common carotid artery, and cardio-ankle vascular index decrease. The apolipoprotein E2 genotype might also contribute to increased risk of cardiovascular complications in subjects with acromegaly, since it is synchronously present with increased IMT and C-reactive protein.

Hypertension remains though the most important mortality prognostic factor in acromegaly. Alterations in reninangiotensin, increased plasma volume (due to sodium retention), increased vascular resistance, and insulin resistance seem to all play a role in the pathogenesis of hypertension [4]. In contrast to diabetes, which resolves in up to two-thirds of patients after remission, hypertension persists in most cases.

Despite studies documenting an increased prevalence of atherosclerotic and vascular risk factors in acromegaly, information is still limited how to best evaluate these patients and predict their risks. Should we assess all patients with FS and AS? The authors previously indicated increased CAD in $41 \%$ of acromegalic patients, regardless of disease control. However, not all studies have determined the utility of either of these scores in predicting nonlethal and lethal ICE. Furthermore, AS seems to be influenced by disease duration and, consequently, occurrence of accompanying metabolic disorders, which are linked to disease control.

The most recent guidelines [5] recommend routine assessments at diagnosis: electrocardiogram, echocardiogram, blood pressure, sleep study, as well as evaluation of the peripheral arterial system. Based on the relatively low number of lethal ICE in the present study [1], it is difficult to conclude that all acromegalic patients older than 40 years of age would benefit from coronary CT. Nevertheless, the value of AS seems of interest in selected patients.

We recommend strict control of clinical and metabolic parameters, which influence FS, as well as that of acromegaly. Further prospective studies are required to assess prognostic implications in the long-term CAD risks and to clarify the necessity to evaluate AS in day-to-day clinical practice, especially in acromegaly patients with wellknown cardiovascular complications. If the partially reversible effect of GH/IGF-I on CAD will be confirmed, this would be an important additional consideration for advocating aggressive lowering of GH/IGF-I levels to reduce the risk of ICE. However, as with all other complications, these prospects are critically dependent on early diagnosis before irreversible changes. The clinical impact of acromegaly treatment on CAD risk is still unclear. A recent study found, for example, an increased cardiovascular risk in patients at 12 months after first-line somatostatin analog treatment, but not in patients after first-line surgery [10].

Targeting normal GH and IGF-1 and optimal management of all traditional cardiovascular risk factors are paramount. The choice of treatment and/or specific targets for hypertension, hyperlipidemia, and diabetes seem to be similar to other high-risk CAD patients without acromegaly, but further studies are clearly needed.

Early diagnosis of acromegaly and effective individualized treatment should be within our reach in the near future.

Conflict of interest The author has no conflicts of interest to declare in connection with this editorial.

\section{References}

1. Ragonese, M., Alibrandi, A., Di Bella, G., et al., Cardiovascular events in acromegaly: distinct role of Agatston and Framingham score in the 5-year prediction. Endocrine (2013). doi:10.1007/ s12020-013-0115-8

2. S. Bonadonna, M. Doga, M. Gola, G. Mazziotti, A. Giustina, Diagnosis and treatment of acromegaly and its complications: consensus guidelines. J. Endocrinol. Invest. 28, $43-47$ (2005)

3. A. Colao, D. Ferone, P. Marzullo, G. Lombardi, Systemic complications of acromegaly: epidemiology, pathogenesis, and management. Endocr. Rev. 25, 102-152 (2004)

4. S. Melmed, Acromegaly pathogenesis and treatment. J. Clin. Investig. 119, 3189-3202 (2009)

5. S. Melmed, F.F. Casanueva, A. Klibanski et al., A consensus on the diagnosis and treatment of acromegaly complications. Pituitary 16, 294-302 (2013)

6. A. Colao, R. Pivonello, L.F. Grasso et al., Determinants of cardiac disease in newly diagnosed patients with acromegaly: results of a 10 year survey study. Eur. J. Endocrinol. 165, 713-721 (2011)

7. L.F. Grasso, A. Colao, Left ventricular synchronicity in acromegaly. Endocrine 44, 1-2 (2013)

8. L. Boero, M. Manavela, T. Merono et al., GH levels and insulin sensitivity are differently associated with biomarkers of cardiovascular disease in active acromegaly. Clin. Endocrinol. (Oxf) 77, 579-585 (2012)

9. P. Anagnostis, Z.A. Efstathiadou, S. Gougoura et al., Oxidative stress and reduced antioxidative status, along with endothelial dysfunction in acromegaly. Hormone and metabolic research $=$ Hormon- und Stoffwechselforschung $=$ Hormones et metabolisme 45 , 314-318 (2013)

10. C. Berg, S. Petersenn, M. Walensi, et al., Cardiac risk in patients with treatment naive, first-line medically controlled and first-line surgically cured acromegaly in comparison to matched data from the general population. Experimental and clinical endocrinology and diabetes : official journal, German Society of Endocrinology [and] German Diabetes Association 121, 125-132 (2013) 